\title{
Construction of Unitary Space-Time Constellations in The Interleaving Sight
}

\author{
Hui $\mathrm{Ji}^{* \dagger}$, Ju Liu*, Dapeng Zhang*, Guowei Zhang* and Yuxi Liu* \\ *School of Information Science and Engineering, Shandong University, Jinan, China 250100 \\ ${ }^{\dagger}$ State Key Lab. of Integrated Services Networks, Xidian University, Xi’an, China 710071
}

Email: jihui016@mail.sdu.edu.cn, juliu@sdu.edu.cn,dpzhang@sdu.edu.cn, zgw415@yahoo.com.cn, liouyuxisimon@163.com

\begin{abstract}
Unitary space-time modulation (USTM) is ideally suited for rapid fading scenarios, where neither the transmitter nor the receiver have the channel state information (CSI). However, it has received limited attention compared with the space-time coding schemes that need CSI. An important reason for that is USTM needs too large calculation amount when meeting with a large size constellation. We propose a size-reduced method for unitary space-time constellations in the sight of interleaving. Resorting to the UST (Unitary Space-Time) symbolwise interleaving, we give a pseudo-random fading effect to the transmitted signals for the receiver to meet the channel requirement in theory. Simulation results show that compared with existing almost unachievable schemes, our method can greatly reduce the complexity of calculation only with a slight penalty in theory.
\end{abstract}

Index Terms-MIMO, USTM, UST symbol, Unitary SpaceTime Constellations, Interleave

\section{INTRODUCTION}

Nowadays, there is a steady increase in people's demands for high data rate and high reliability wireless communication services. Increasing the channel bandwidth is an intuitive and simple remedy, however, it would be ultimately unsatisfactory. A more feasible solution is to utilize space-time processing techniques with multiple antennas at the transmitters and/or at the receivers.

The space-time coding techniques meet its burst since 1990s, based on the research of MIMO (Multiple Input Multiple Output) channel capacity [1]-[3]. Depending on whether the channel state information (CSI) is needed, space-time codes can be classified into two types: type I that needs CSI and type II that doesn't need. Type I includes layered spacetime code (BLAST) [4], space-time trellis code (STTC) [5] and space-time block code (STBC) [6], [7]. Type II includes differential space-time code (DSTC) [8] and unitary space time modulation (USTM) [9]. Most researchers concentrate on type I codes which need perfect CSI at least at the receivers. However, estimating the fading coefficients between each pair of transmit and receive antenna elements becomes increasingly difficult as either the fading rate or number of antennas increases. In some extreme conditions, it would be impossible to learn even a single coefficient between one transmitter and one receiver antennas, which limits the application of type I space-time codes.

A constellation reduction scheme (UST-RC) has been proposed in [10]. The scheme can only deal with the situation that

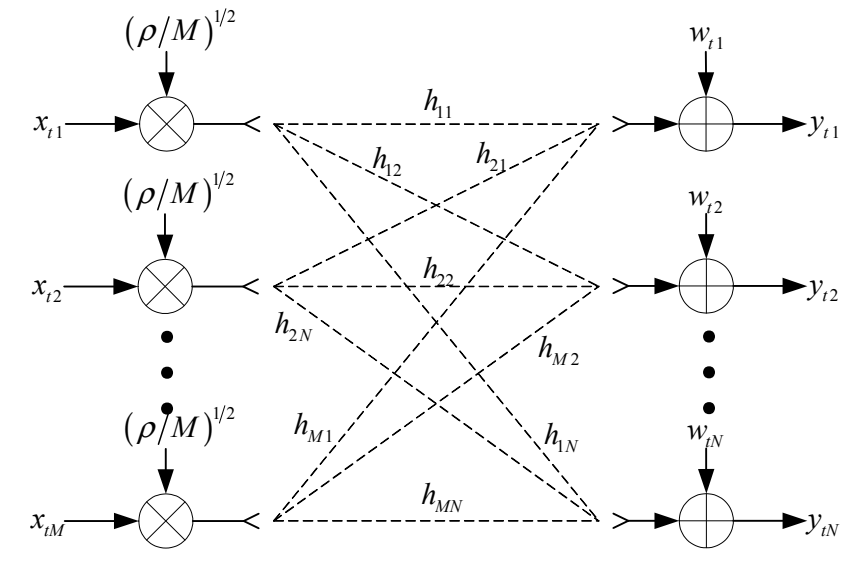

Fig. 1. MIMO system comprising $M$ transmitter and $N$ receiver antennas.

the coherence interval $T$ is a composite number. In this paper, we propose a generalized size reduced constellation unitary space-time modulation scheme which has no limitation on $T$. It suits for scenarios that $T$ can be both composite and prime numbers. Although this method is suboptimal, it can deliver the modulation into practice from theory.

The rest of this paper is organized as follows. Section II reviews the derivation and development of unitary space-time modulation briefly. Section III describes our proposed scheme: the system of unitary space-time modulation with reduced constellations in the sight of interleaving. In section IV, we analyse the feasibility of the scheme and the differences of several reduction schemes. Finally, we conclude this paper in section V.

\section{Unitary SPace-Time Modulation}

The idea of USTM is proposed by Marzetta and Hochwald from calculating the capacity of fast block fading channels [3], where the fading coefficients are constant for $T$ symbol periods, after which it changes to another independent value. All of the transmitters and the receivers don't know the fading coefficients.

Fig. 1 displays a wireless communication system equipped with $M$ transmitter antennas and $N$ receiver antennas. The received signal can be written in matrix form as

$$
\boldsymbol{Y}=\sqrt{\frac{\rho}{M}} \boldsymbol{X} \boldsymbol{H}+\boldsymbol{W}
$$


where $\boldsymbol{X}$ is the $T \times M$ matrix of transmitted signals, $\boldsymbol{Y}$ is the $T \times N$ matrix of received signals. $\boldsymbol{H}$ is the $M \times N$ matrix of propagation coefficients with every component $\mathcal{C N}(0,1)$ distributed. $\boldsymbol{W}$ is the $T \times N$ matrix of additive noise, whose entries are independent and identically $\mathcal{C N}(0,1)$ distributed.

It is clear that

$$
E\{\boldsymbol{Y} \mid \boldsymbol{X}\}=\mathbf{0},
$$

and

$$
E\left\{\boldsymbol{Y} \boldsymbol{Y}^{\dagger} \mid \boldsymbol{X}\right\}=\boldsymbol{I}_{T}+\frac{\rho}{M} \boldsymbol{X} \boldsymbol{X}^{\dagger}
$$

The probability density function of the received signal conditioned on the transmitted signal can be depicted by

$$
p(\boldsymbol{Y} \mid \boldsymbol{X})=\frac{\exp \left(-\operatorname{tr}\left\{\left[\boldsymbol{I}_{T}+(\rho / M) \boldsymbol{X} \boldsymbol{X}^{\dagger}\right]^{-1} \boldsymbol{Y} \boldsymbol{Y}^{\dagger}\right\}\right)}{\pi^{T N} \operatorname{det}^{N}\left[\boldsymbol{I}_{T}+(\rho / M) \boldsymbol{X} \boldsymbol{X}^{\dagger}\right]}
$$

where the superscript $\dagger$ denotes "conjugate transpose", $\boldsymbol{I}_{T}$ denotes the $T \times T$ identity matrix and "tr" denotes "trace". It has been shown in [3] that, a capacity achieving signal should have the structure of $\boldsymbol{X}=\boldsymbol{\Phi} \boldsymbol{V}=\left[v_{1} \phi_{1}, v_{2} \phi_{2}, \cdots, v_{M} \phi_{M}\right]$, where $\boldsymbol{\Phi}$ is a $T \times T$ isotropically distributed unitary matrix, and $\boldsymbol{V}$ is an independent $T \times M$ real, nonnegative, diagonal matrix. Further more, as $T$ increases, the value of every component in $\boldsymbol{V}$ approaches $\sqrt{T}$ with a very high probability. After further investigation, [9] first gave the definition of USTM: when either $T \gg M$ or $\rho$ is large and $T>M$, to achieve capacity, the transmitted signal matrix with $R$ bits per channel use has the partitioned form

$$
\boldsymbol{X}_{l}=\sqrt{T} \boldsymbol{\Phi}_{l}, l=0, \cdots, L-1
$$

where $\boldsymbol{\Phi}_{l}$ is a $T \times M$ unitary matrix and the constellation size is $L=2^{T R}$. The methods for systematic design of unitary space-time signals can be found in [11] which gave a metric for the performance of unitary constellations:

$$
\delta=\max _{l=1, \cdots, L-1} \operatorname{tr}\left(\boldsymbol{\Phi}_{l}^{\dagger} \boldsymbol{\Phi}_{0} \boldsymbol{\Phi}_{0}^{\dagger} \boldsymbol{\Phi}_{l}\right)
$$

The smaller the value of $\delta$ is, the better the performance would be. The $M$ columns of $\boldsymbol{\Phi}$ which represent the temporal signals fed into the transmitter antennas are mutually orthogonal. The received symbol can be demodulated by maximum-likelihood (ML) receiver [9]:

$$
\begin{aligned}
\boldsymbol{\Phi}_{\mathrm{ml}} & =\arg \max _{\mathbf{\Phi}_{l} \in\left\{\boldsymbol{\Phi}_{0}, \cdots, \boldsymbol{\Phi}_{L-1}\right\}} p\left(\boldsymbol{Y} \mid \boldsymbol{\Phi}_{l}\right) \\
& =\arg \max _{\boldsymbol{\Phi}_{l} \in\left\{\boldsymbol{\Phi}_{0}, \cdots, \boldsymbol{\Phi}_{L-1}\right\}} \operatorname{tr}\left(\boldsymbol{Y}^{\dagger} \boldsymbol{\Phi}_{l} \boldsymbol{\Phi}_{l}^{\dagger} \boldsymbol{Y}\right)
\end{aligned}
$$

Although it is possible to use USTM for wireless communication in fast fading channels, in situations where the value of coherence time $T$ is relatively large, the original systematic constellation design scheme [11] meets with two severe problems. First, given a value of coherence time $T$, it is hard to find an optimal constellation for communication. The most trustworthy method is exhaustive search, however, this is not realizable even when $T=8$. Second, for a large value of

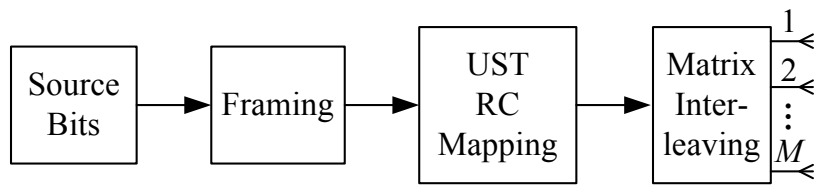

Fig. 2. Block diagram of USTM system with reduced constellations.

$T$, the amount of computation is too large for the receiver to realize. For example, when $T=32$ and $R=1$ bit per channel use, to demodulate every block of $T$ bits, the receiver has to compute every $\operatorname{tr}\left(\boldsymbol{Y}^{\dagger} \boldsymbol{\Phi}_{l} \boldsymbol{\Phi}_{l}^{\dagger} \boldsymbol{Y}\right)$, where $l=0, \cdots, L-1$, and $L=2^{T R}=2^{32}$. This is almost impossible in reality. Existing investigations for these problems need too deep mathematical theory and is not easy to be realized.

\section{Unitary Space-Time Modulation with Reduced CONSTELLATIONS}

\section{A. Modulation}

Motivated by the procedure that interleaving can be realized symbol by symbol, we utilize signal matrix level interleaving to give a simple method which can easily reduce the unitary space-time signal constellations.

The block diagram of USTM system with reduced constellation (UST-RC) is given in Fig. 2. Source bits are partitioned into frames, where the number of signals in one frame is equal to the real channel coherence time $T$. Every frame is divided into several blocks. where $T$ is not required to be a composite number. If $T$ is prime the frame can be divided unequally. For example $T=7$ and $R=1$, we can use double constellation sets with $T_{c 1}=4$ and $T_{c 2}=3$. Then, we map the first 4 bits in a block into a $T_{c 1} \times M$ UST matrix and map the other 3 bits into a $T_{c 2} \times M$ UST matrix.

Classical unitary space-time modulation requires that signals in one frame should be considered as a whole part to fit the Rayleigh flat fading channel. Here, we use $T_{c}$ to construct constellation. Here, $T_{c}$ denotes the pseudo coherence time, which is appeared before as $T_{c 1}$ and $T_{c 2}$. Then, the constellation has $L=2^{T_{c} R}$ unitary space-time signals, whose size is only $1 / 2^{\left(T-T_{c}\right) R}$ of its original counterpart. It is easy to learn that every block corresponds to a UST signal. The interleaver in Fig. 4 scrambles the UST signals which would be transmitted. When using this reduced constellation, we must always keep in mind that the number of transmitted antennas $M$ should be smaller than the minimum of $T_{c}$.

Here, we should note that the transmitted signal matrix in USTM [9] which is $T \times M$ dimensional is a little different from the capacity achieving form [3] which is $T \times T$ dimensional. In other words, [3] told us if the number of transmitter antennas $M$ is larger than $T$, the channel capacity is just the same as with $T$ transmitter antennas. However, when employing USTM, the number of transmitter antennas must be smaller than the value of coherence interval, namely, $M<T$. As we know, the effect of the transmitted signal $\boldsymbol{X}$ on the conditional probability density of the received signal is through the product 


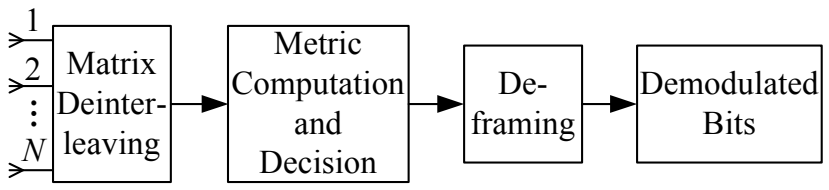

Fig. 3. Demodulation of RC-USTM system.

$\boldsymbol{X} \boldsymbol{X}^{\dagger}$. So, if $M=T$, the product of $\boldsymbol{X}$ and $\boldsymbol{X}^{\dagger}$ would be $\boldsymbol{I}_{T}$, which means that the transmitted signal carries no information. Therefore, in our scheme, the number of transmitter antenna $M$ should be smaller than the minimal value of coherence intervals in constellation $\min \left(T_{c}\right)$.

\section{B. Demodulation}

Through an $M \times N$ Rayleigh flat fading MIMO channel, interleaved signals arrive at the receiver. Because $\boldsymbol{X}=\sqrt{T_{c}} \boldsymbol{\Phi}$, the conditional probability density is

$$
p(\boldsymbol{Y} \mid \boldsymbol{\Phi})=\frac{\exp \left(-\operatorname{tr}\left\{\left[I_{T_{c}}-\frac{1}{1+M /\left(\rho T_{c}\right)} \boldsymbol{\Phi} \boldsymbol{\Phi}^{\dagger}\right] \boldsymbol{Y} \boldsymbol{Y}^{\dagger}\right\}\right)}{\pi^{T_{c} N} \operatorname{det}^{N}\left[I_{T_{c}}+\left(\rho T_{c} / M\right) \boldsymbol{\Phi} \boldsymbol{\Phi}^{\dagger}\right]}
$$

If we view the interleaver in the transmit, the channel and the deinterleaver in the receiver as a whole, we can see that the channel of the system has changed from a relatively slower fading into a pseudo-fast fading. In other words, signal matrix level interleaver and deinterleaver give a fast fading effect for signals $\boldsymbol{X}$ to be demodulated. The demodulation procedure can be done using (5), where the received signal $\boldsymbol{Y}$ is $T_{c} \times N$ dimensional.

The upper figure in Fig. 4 demonstrates the construction of signals in $J$ frame before interleaving, where every block is composed of $T_{c} \times M$ signals. Here, we suppose that the signal of one frame is divided into 2 blocks, and their sizes can be the same, but also can be different. These blocks are interleaved with blocks in other frames. The lower figure in Fig. 4 gives the structure of data blocks after interleaving. Indices indicate the blocks that participate in the interleaving procedure and $f(\cdot)$ is the permutation function for interleaving. The receiver has the information of $f(\cdot)$.

If we want to demodulate signals in a real coherence time $T$, using classical maximum likelihood method proposed by [9], we have to compute (5) for $L=2^{T R}$ times. By employing the reduced scheme, the maximum likelihood decoding can be directly derived:

$$
\mathbf{\Phi}_{\mathrm{ml}}=\arg \max _{\mathbf{\Phi}_{l} \in\left\{\boldsymbol{\Phi}_{0}, \cdots, \mathbf{\Phi}_{2} T_{c} R_{-1}\right\}} \operatorname{tr}\left(\boldsymbol{Y}^{\dagger} \boldsymbol{\Phi}_{l} \boldsymbol{\Phi}_{l}^{\dagger} \boldsymbol{Y}\right)
$$

Here, to get the same number of demodulated signals, the calculation amount is reduced exponentially. We must notice that here $\boldsymbol{Y}$ is $T_{c} \times N$ dimensional and $\boldsymbol{\Phi}$ is $T_{c} \times M$ dimensional respectively. If taking into account the sizes of matrices in calculating the traces, compared with the original method, our scheme should be even simpler.

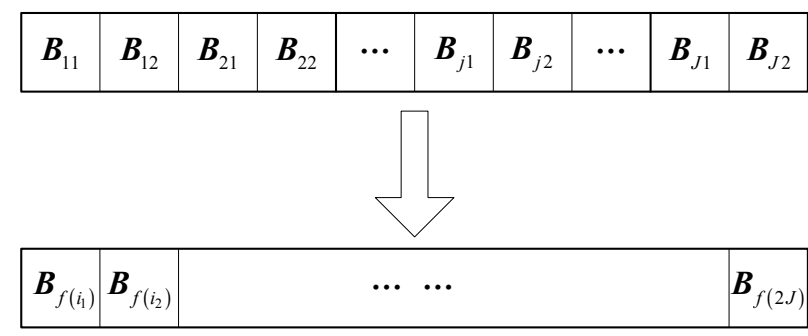

Fig. 4. Interleaving for signals, where the signals in different constellations are permuted together.

\section{Simulation Results AND Analysis}

\section{A. The feasibility of RC-USTM}

For simplicity, we always assume in simulations that the channel operates with $R=1$ bit per channel use. $T$ and $T_{c}$ denote the real coherence time and the pseudo coherence time in reduced constellation respectively. The first simulation is to exemplify that the proposed scheme can really be put into use and can give transmit diversity.

In this simulation, we assume the real coherence time is $T_{r}=31$, in which situation, it is impossible for engineering with so large constellation using classic method. If we use the CSI-needed space-time codes, training symbols would take a large part of the coherence interval. Here, every frame is divided into 4 blocks. In 3 blocks $T_{c_{1}}=8$ and in another block $T_{c_{2}}=7$. Here, we use the random interleaving. Analyzing the differences of different kinds of interleaving is beyond our domains. The reduced constellation is generated by using $u=[220,191,6,87,219,236,173,170]$ for $T_{c_{1}}=8$, and $u=$ $[1,2,7,11,24,55,101]$ for $T_{c_{2}}=7$. $\boldsymbol{\Phi}_{0}$ is a $T_{c} \times M$ matrix whose columns are the first $M$ columns of a DFT matrix [11] (The elements of $u$ may not be the best for this situation, however, how to find the optimal one is another topic and our aim in this simulation is just to show the feasibility of the proposed scheme). If using the constellation for $T$, the signal number in constellation is $2^{T R}=2^{32}$, which is almost impossible to be used in reality and can not be implemented by simulation. Here, the proposed scheme employs $T_{c}=8$ which lead to a reduced constellation with a signal number $2^{T_{c} R}=$ $2^{8}$. It is easy to see that the size of the reduced constellation is only $2^{T_{c} R} / 2^{T R}=1 / 2^{24}$ of the original constellation size. Fig. 5 shows that the proposed scheme can actually be put into use.

\section{B. The roles of $T$ and $T_{c}$}

In this experiment, we try to find whether $T$ or $T_{c}$ has more effects on the performance of USTM, and try to discover how the values of $T_{c}$ affect the results. We consider $T=7$ condition. Each $T=7$ frame is divided into two blocks with $T_{c 1}=4$ and $T_{c 2}=3$. We set $J=10$, which means that there are $2 \times J=20$ blocks take part in interleaving. By exhaustive search, we find the best $u$ for $T=7,4$ and 3 are $[1,2,7,11,24,55,101],[1,3,4,8]$ and $[1,2,4]$ respectively.

In theory, a constellation with a larger $T$ can perform better when other parameters are all the same. However, as 


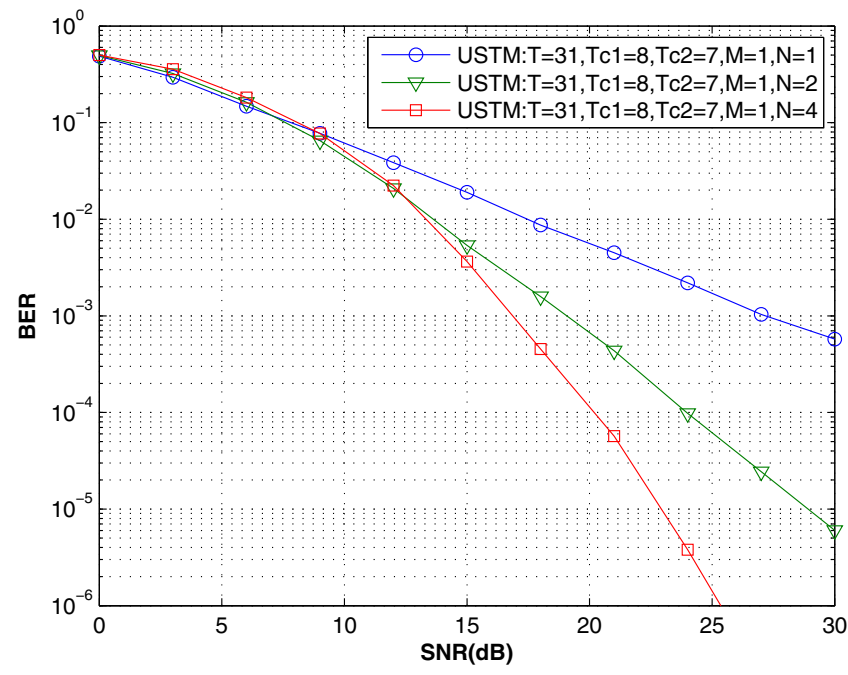

Fig. 5. Interleaving for signals, where the signals in different constellations are permuted together.

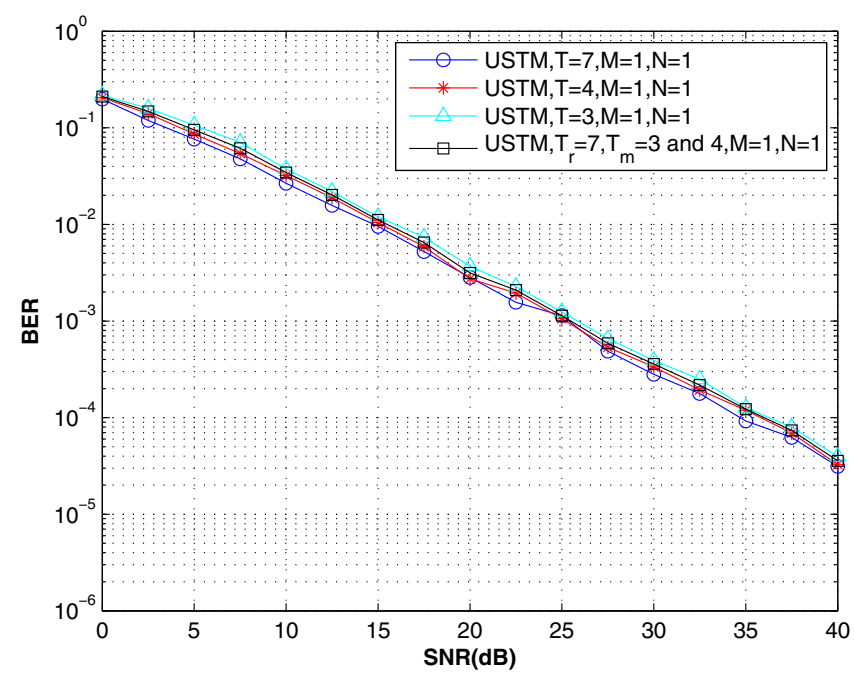

Fig. 6. Interleaving for signals, where the signals in different constellations are permuted together.

the constellation design is now still an open problem, the constellation construction with larger $T$ can not insure a better performance compared with a smaller $T$.

From Fig. 6 the differences between the three curves corresponding to $T=7,4$ and 3 are very little. The performance of the proposed scheme is only a little inferior to $T=4$ and is better than $T=3$. As we know, the good BER performances of USTM are achieved when SNR are high enough. In engineering, people must combine it with error control coding technique to save the transmit power. And then, the BER performances of these constellations are negligible, and the proposed scheme can reduce the calculation complexity and storage requirement for a large scale.

\section{CONClusion}

In this paper, we have proposed a size-reduced constellation scheme for unitary space-time modulation. We employed signal matrix level interleaving to give a pseudo-random fading effect for the receiver, and we showed by simulations that this scheme can actually be put into use, only with a slight performance punishment in theory. We also demonstrated that the constellation size, rather than the real coherence time, can affect the performance. When $T$ is given, there may be several values that can be selected for $T_{c}$. If selecting a smaller one, it is easier to implement, and if selecting a larger one, the system would give a better diversity potentiality because both the pseudo coherence time $T_{c}$ and the value of $M$ can be larger. We have dealt with the conditions that the real coherence time $T$ can be both a composite number and a prime number.

\section{ACKNOWLEDGMENT}

This work was supported in partial by the National Natural Science Fund of China (60872024), the Natural Science Fund of Shandong Province (Y2007G04), the Cultivation Fund of the Key Scientific and Technical Innovation Project(708059), Ministry of Education of China, the open research Fund of National Mobile Communications Research Laboratory in Southeast University (No.W200802) and the State Key Lab. of Integrated Services Networks in Xidian University(ISN-9-03).

\section{REFERENCES}

[1] E. Telatar, "Capacity of multi-antenna Gaussian channel," Tech. Rep., AT\&T Bell Labs., 1995.

[2] G. J. Foschini and M. J. Gans, "On limits of wireless communication fading environment when using multiple antennas," Wireless Personal Commun., vol. 6, pp. 311-335, 1998.

[3] T. L. Marzetta and B. M. Hochwald, "Capacity of a mobile multipleantenna communication link in Rayleigh flat fading," IEEE Trans. Inform. Theory, vol. 45, No. 1, pp. 139-157, Jan. 1999.

[4] G. J. Foschini, "Layered space-time architecture for wireless communication in a fading environment when using multiple antennas," Bell Labs Technical Journal, vol. 1, pp. 41-59, 1996.

[5] V. Tarokh, N. Seshadri and A. R. Calderbank, "Space-time codes for high data rate wireless communication: performance criterion and code construction," IEEE Trans. Inform. Theory, vol. 44, No. 2, pp. 744-765, Mar. 1998.

[6] S. M. Alamouti, "A simple transmit diversity technique for wireless communications," IEEE J. Select. Areas Commun., vol. 16, No. 8, pp. 1451-1458, Oct. 1998.

[7] V. Tarokh, H. Jafarkhani and A. R. Calderbank, "Space-time block codes from orthogonal designs," IEEE Trans. Inform. Theory, vol. 45, No. 5, pp. 1456-1467, Jul. 1999.

[8] H. Jafarkhani and V. Tarokh,"Multiple transmit antenna differential detection from generalized orthogonal designs," IEEE Trans. Inform. Theory, vol. 47, No. 6, pp. 2626-2631, Sep. 2001.

[9] B. M. Hochwald and T. L. Marzetta, "Unitary space-time modulation for multiple-antenna communications in Rayleigh flat fading," IEEE Trans. Inform. Theory, vol. 46, No. 2, pp. 543-564, Mar. 2000.

[10] D. Zhang, J. Liu, and H Ji, "A simple size-reduced constellation scheme for unitary space-time modulation," in Proc. IEEE Singapore International Conference on Communication Systems (ICCS' 2008), Guangzhou, China, Nov. 2008, pp. 87-91.

[11] B. M. Hochwald, T. L. Marzetta, T. J. Richardson, W. Sweldens, and R. Urbanke, "Systematic design of unitary space-time constellations," IEEE Trans. Inform. Theory, vol. 46, No. 6, pp. 1962-1973, Sept. 2000. 